\title{
BRM270 Inhibits the Proliferation of CD44 Positive Pancreatic Ductal Adenocarcinoma Cells via Downregulation of Sonic Hedgehog Signaling
}

\author{
Do Luong Huynh, ${ }^{1}$ Hyebin Koh, ${ }^{1}$ Nisansala Chandimali, ${ }^{1}$ Jiao Jiao Zhang, ${ }^{1,2}$ \\ Nameun Kim, ${ }^{1,3}$ Tae Yoon Kang, ${ }^{1}$ Mrinmoy Ghosh, ${ }^{1,4}$ Meeta Gera, ${ }^{1}$ Yang-Ho Park, \\ Taeho Kwon $\mathbb{D}^{1,6}$ and Dong Kee Jeong $\mathbb{D}^{1}$ \\ ${ }^{1}$ Laboratory of Animal Genetic Engineering and Stem Cell Biology, Advanced Convergence Technology \& Science, \\ Jeju National University, Jeju 63243, Republic of Korea \\ ${ }^{2}$ Chongqing Key Laboratory of Forage and Herbivore, College of Animal Science and Technology, \\ Southwest University, Chongqing 400715, China \\ ${ }^{3}$ Jeju Special Self-Governing Province Livestock Promotion Agency, 13 Chuksanmaeul-gil, Jeju 63078, Republic of Korea \\ ${ }^{4}$ Department of Biotechnology, Division of Research and Development, Lovely Professional University, Punjab 144411, India \\ ${ }^{5}$ BRM Institute, Seoul 01756, Republic of Korea \\ ${ }^{6}$ Primate Resource Center, Korea Research Institute of Bioscience and Biotechnology (KRIBB), \\ Jeongeup-si, Jeonbuk 56216, Republic of Korea
}

Correspondence should be addressed to Taeho Kwon; taehokwonk@gmail.com and Dong Kee Jeong; newdkjeong@gmail.com

Received 7 August 2018; Revised 26 November 2018; Accepted 16 January 2019; Published 24 February 2019

Academic Editor: Sakthivel Muniyan

Copyright (C) 2019 Do Luong Huynh et al. This is an open access article distributed under the Creative Commons Attribution License, which permits unrestricted use, distribution, and reproduction in any medium, provided the original work is properly cited.

Pancreatic cancer has a poor survival rate as compared to other types of cancer. Surface marker CD44 plays important role in epithelial-mesenchymal transition and cancer stem cell phenotype. Therefore, targeting CD44 positive pancreatic cancer cells might enhance therapies effectiveness. Our previous studies indicated the antitumorigenesis effect of BRM270 in osteosarcoma, lung cancer, and glioblastoma; however there is no evidence on BRM270 impacts on pancreatic cancer growth. In this study, we investigated the effect of BRM270 on the isolated CD44 positive pancreatic ductal adenocarcinoma cells (CD44 ${ }^{+}$PDAC). Results showed that CD44 positive cells undergo apoptosis induced by BRM270. Moreover, BRM270 also inhibits stemness and metastasis traits in $\mathrm{CD} 44^{+}$PDAC via Sonic hedgehog signaling pathway and SALL4 expression. In vivo study indicated that tumor growth derived from CD $44^{+}$PDAC was suppressed as daily uptake by BRM270 $5 \mathrm{mg} / \mathrm{kg}$. These data suggest the alternative approach in antipancreatic tumorigenesis via herbal plants extract and selectively targeting CD $44^{+}$PDAC cells in tumor.

\section{Introduction}

Survival rate in pancreatic cancer is extremely lower as compared to other cancers $[1,2]$. Pancreatic ductal adenocarcinoma (PDAC) accounts for $80 \%$ of pancreatic cancer and becomes one of the most death cases in the world $[3,4]$. Many attempts to cure PDAC have been deployed. However, therapeutic efficiency remains low, due to its silent symptoms, lack of early diagnosis, or effective therapies $[3,4]$.
Many evidences indicated that CD44 expression is strongly associated with epithelial-mesenchymal transition (EMT) and cancer stem cell (CSC) phenotypes such as tumor invasion, metastasis, recurrence, or chemoresistance [5]. CD44 is also considered as one of the CSC markers in various types of tumors $[5,6]$. Furthermore, clinical reports showed that high expression of CD44 is linked to poor survival rate [6]. Therefore, new findings in anticancer cells targeting high CD44 expression could be a promising approach $[6,7]$. 
Sonic hedgehog (Shh) signaling pathway plays important role in tumorigenesis, including tumor initiation, promotion or metastases in skin, leukemia, lung, brain, and gastrointestinal cancers [8]. In pancreatic cancer, evidences also indicated that Shh signaling pathway regulates tightly pancreatic CSCs stemness and metastatic traits $[9,10]$. Many target genes such as transcription factors of pluripotency (Oct-4, Sox-2, Nanog, and $\mathrm{c}-\mathrm{Myc}$ ) or epithelial-mesenchymal transition (EMT) genes (MMP-9, CXCR4, Snail-1, and N-cad) are upregulated via Shh stimulation, resulting in high metastatic phenotype and drug resistance or tumor relapse as well [11,12]. Therefore, the inhibition of Shh signaling becomes the main concern in antipancreatic cancer.

BRM270 in previous studies shows its effects in antitumorigenesis [13-15]. As a natural extract, BRM270 inhibits the proliferation of lung adenocarcinoma and glioblastoma stem cells in vitro and in vivo. In this study we aim to examine the effects of BRM270 on CD44 positive $\left(\mathrm{CD} 44^{+}\right)$ PDAC cells. BRM270 significantly represses the proliferation and metastasis and deactivates SALL4-mediated CSCs maintenance and Shh expression and activities, thus favoring antipancreatic tumor treatment. Taken together, BRM270 might be a multitarget arrow in cancer treatment, targeting both in CSCs maintenance and metastasis phenotype.

\section{Materials and Methods}

2.1. Reagents. BRM270 supplied by BRM Institute (Seoul, Korea) was extracted by methanol/ethanol, followed by rotary concentration. Pellet was dissolved in DMSO (SigmaAldrich, MO, USA) and stored at $-20^{\circ} \mathrm{C}$ for further analysis. Polyclonal antibodies against Bcl-2, Bcl-xL, Caspase-3, CD133, CD44, c-Myc, CXCR4, p53, GADPH, Gli-1, Nanog, N-cadherin, Oct-4, SALL-4, Shh, Snail-1, Sox-2 antibodies, and horseradish peroxidase- (HRP-) conjugated anti-rabbit or anti-mouse IgG were purchased from Santa Cruz Biotechnology (Santa Cruz, CA, USA).

2.2. Isolation of CD44 Positive PDAC Cells. Cells in log phase were used for magnetic-activation cell sorting (MACS) separation with CD44 microbeads (Miltenyi Biotec, Germany) as manufacturer's protocol. Subpopulations of CD44 negative $\left(\mathrm{CD} 44^{-}\right)$and $\mathrm{CD} 44$ positive $\left(\mathrm{CD} 44^{+}\right)$were subjected to further analysis.

2.3. Cell Culture. PANC-1 and BxPC-3 cells were from cultured DMEM (Gibco, CA, US), supplemented with 10\% FBS (Welgene, Korea) and 1\% antimycotic type and maintained in a humidified atmosphere of $5 \% \mathrm{CO}_{2}$ in incubator at $37^{\circ} \mathrm{C}$, two passages weekly. CSCs after isolation by magnetic activated cells sorting (MACS, Miltenyi Biotec, Germany) were maintained in DMEM/F12 plus with 2\% B27, $10 \mathrm{ng} / \mathrm{mL}$ hEGF, (Sigma-Aldrich, MO, US), and $10 \mathrm{ng} / \mathrm{mL}$ bFGF (KOMA Biotech, Seoul, Korea) [16].

2.4. Flow Cytometry Assay. Cells after isolation by MACS were certified by CD44-APC (Miltenyi Biotec, Germany)
FACS analysis as manufacturer's protocol. For apoptosis assay, in brief cells after $48 \mathrm{~h}$ treatment by BRM270 were resuspended in $100 \mu \mathrm{l}$ binding buffer containing $5 \mu$ l Annexin V-FITC conjugated antibody and $5 \mu \mathrm{l}$ propidium iodide for exactly $10 \mathrm{~min}$ in the dark at room temperature. Cells were then analyzed on BD Accuri C6 cytometer (BD Biosciences, NJ, US).

2.5. Cell Viability. $5 \times 10^{3}$ cells were seeded into a 96-well plate and incubated $24 \mathrm{~h}$ before treatment with or without indicated BRM270 concentrations. After 48 h, cell cytotoxic effects were measured by EZ-Cytox kit (Daeil Lab, Seoul, Korea) according to the manufacturer's protocol. The cell viability results are presented as the ratio of optical density at $450 \mathrm{~nm}\left(\mathrm{OD}_{450}\right)$ that was calculated using the following formula: $(\%)$ cell viability $=(\mathrm{OD}$ treatment groups or control groups/OD vehicle control group) $\times 100 \%$.

2.6. Immunocytochemistry Staining. Cells were fixed by $3.7 \%$ paraformaldehyde (PFA). Before overnight staining with primary antibodies of interest, cells were blocked by phosphatebuffered saline $0.1 \%$ Tween-20 (PBST 1X) with 3\% BSA. After washing 2 times with PBST 1X, secondary antibodies were added and followed by $2 \mathrm{~h}$ incubation. After two washes by PBST $1 \mathrm{X}$, cells were stained $10 \mathrm{~min}$ with $4^{\prime}, 6$-diamidino-2-phenylindole (DAPI) before capturing on microscope.

2.7. Western Blotting. Protein quantity in lysates was determined using BCA assay. Proteins were separated on a $12 \%$ SDS-PAGE, transferred electrophoretically (Bio-Rad, CA, USA) onto a polyvinylidene fluoride (PVDF) membrane, and blocked with $5 \%$ nonfat milk powder (w/v) in PBST 1X for $1 \mathrm{~h}$ at room temperature, followed by incubating with primary antibodies or with anti-GADPH mouse monoclonal antibody as an internal control overnight at $4^{\circ} \mathrm{C}$ and with appropriate HRP-conjugated secondary antibodies at RT for 2 h. The bands were captured by ImageQuant ${ }^{\mathrm{TM}}$ LAS $4000 \mathrm{mini}$ Fujifilm [16].

2.8. Clonogenic Assay. $1 \times 10^{3}$ cells were seeded in 6-well plate at $37^{\circ} \mathrm{C} / 5 \% \mathrm{CO}_{2}$. After 7 days for incubation, cells were fixed with $3.7 \%$ formaldehyde and stained with $0.05 \%$ crystal violet/washing by PBS 1X before capturing [16].

2.9. Sphere Formation. $1 \times 10^{3}$ cells were seeded into ultralow attachment 6-well plate (Corning, NY, US) in DMEM/F12 plus with 2\% B27, $10 \mathrm{ng} / \mathrm{mL}$ hEGF (Sigma-Aldrich, MO, US), and $10 \mathrm{ng} / \mathrm{mL}$ bFGF (KOMA biotech, Seoul, Korea) with and without BRM270 treatment. After 7 days of incubation, spheres were captured by microscope.

2.10. In Vitro Cell Migration and Invasion Assays. Cell migration assay was performed using $8-\mu \mathrm{m}$ pore size hanging cellinserts (Merck Millipore, MA, US). $1 \times 10^{5}$ cells in $0.5 \%$ FBSDMEM were seeded in upper chamber while lower chamber was filled with $20 \%$ FBS. After $48 \mathrm{~h}$ incubation, migrating 
cells were stained with $0.05 \%$ crystal violet $(\mathrm{w} / \mathrm{v})$. The number of migrated cells on the lower surface of the membrane was counted under a microscope in five random fields at $100 \times$. For cell invasion assay, all procedures were carried out as in the migration assay, except that Matrigel matrix growth factor reduced basement (BD Biosciences, NJ, US) $(3.5 \mathrm{mg} / \mathrm{mL})$ was coated on the upper chamber according to the manufacturer's protocol.

2.11. Wound Healing Assay. Cells were used in wound healing assay as per manufacturer's protocol. Briefly, cells at log phase were seeded into IncuCyte ImageLock 96-well microplates, reaching 98\%-100\% confluence after overnight. Monolayer of cells was scratched by wound-maker and underwent realtime imaging by IncuCyte system (Essen Bioscience, MI, US) as indicated time points.

2.12. In Vivo Evaluation. Mice were maintained according to a protocol approved by the Institutional Animal Care and Use Committee of Jeju National University (Jeju, Korea). Tumors were induced by subcutaneously injecting $1 \times 10^{6}$ cells in $100 \mu \mathrm{l}$ mixed volume Matrigel (Sigma-Aldrich, MO, USA) and PBS into the flanks of 6-week-old nude male BALB/c-nu mice ( $n=3$ /each group). Tumors were measured every 7 days by using caliper. BRM270 was orally supplied every day at dose of $5 \mathrm{mg} / \mathrm{kg}$. The tumor volume $(\mathrm{V}=\mathrm{W} \times \mathrm{L} \times \mathrm{H} / 2)$ was evaluated by length $(\mathrm{L})$, height $(\mathrm{H})$, and width $(\mathrm{W})$. Mice were sacrificed at day 35 after cell injection.

2.13. Statistical Analysis. Statistical analysis was performed using Graphpad Prism 6.02. Data are expressed as mean \pm standard deviation (SD). Experimental differences were examined using ANOVA and Student's $t$-tests, as appropriate. $P$ values of $<0.05$ were considered to indicate statistical significance.

\section{Results}

3.1. Isolation of CD44 Positive PDAC Cells. It is implied that CD44 surface marker is associated with PDAC malignance [6]. In this study, BxPC-3 and PANC-1 were chosen for MACS separation of the $\mathrm{CD} 44^{+}$cells. Results showed that proportion of CD44 was differed significantly after separation, represented by FACS analysis and Western blotting (Figures $1(\mathrm{a})$ and $1(\mathrm{~b})$ ). Immunocytochemistry staining showed the CD44 expression in tumor spheres of CD $44^{+}$BxPC-3 and PANC-1 (Figure 1(c)). Regarding tumor formation, cells with high expression of CD44 showed the dominance in tumor sizes and weights (Figures $1(\mathrm{~d})$ and $1(\mathrm{e})$ ). These data suggested that $\mathrm{CD}_{4} 4^{+} \mathrm{PDAC}$ cells are more malignant as compared to CD44 negative subpopulation.

3.2. BRM270 Inhibits the In Vitro Malignance of CD44 Positive PDAC Cells. Cells with high expression of CD44 were exposed to BRM270 $50 \mu \mathrm{g} / \mathrm{mL}$. After $48 \mathrm{~h}$ treatment, signs of apoptosis were examined by cell viability assay, FACS Annexin V, and Western blotting. Results showed that BRM270 inhibited the proliferation of CD $44^{+}$PDAC cells dose dependently (Figure 2(a)). FACS analysis showed the increases of apoptotic cells under BRM270 treatment (Figure 2(b)). Furthermore, there were the activation of caspase3 and downregulation of PCNA (Figure 2(c)). BRM270 also decreased the clonogenicity of $\mathrm{CD} 44^{+}$PDAC cells (Figure 2(d)). These data indicated that BRM270 induced the apoptosis of CD $44^{+}$PDAC cells. Moreover, inhibitory effects of BRM270 to CD $44^{+}$PDAC malignances also were noted via cell mobility assay. BRM270 treatment decreased considerably the numbers of cells migrating and invading (Figure 2(e)). Furthermore, BRM270 inhibited notably the wound closures of CD $44^{+}$PDAC after $24 \mathrm{~h}$ treatment (Figure 2(f)). Taken together, these data suggested that BRM270 prevents effectively the in vitro malignances of $\mathrm{CD} 44^{+} \mathrm{PDAC}$ cells.

3.3. BRM270 Represses Self-Renewal Capacity of CD44 Positive $P D A C$ Cells. Self-renewal capacity is driven by various stemness genes such as CD133, SALL4, Oct4, Sox-2, and Nanog $[17,18]$. To elucidate the inhibitory effects of BRM270 on CD $44^{+}$PDAC self-renewal capacity, cells were proceeded to Western blotting, immunocytochemistry staining, and tumor sphere formation. Results showed that there were downregulations of stemness genes, including CD44, CD133, SALL4, Oct4, Sox-2, and Nanog under $50 \mu \mathrm{g} / \mathrm{mL}$ BRM270 treatment (Figure 3(a)). Levels of these genes were repressed in immunocytochemistry staining (Figure 3(b)). Furthermore, tumor sphere formations of $\mathrm{CD} 44^{+}$PDAC cells were inhibited in the presence of $50 \mu \mathrm{g} / \mathrm{mL}$ BRM270 (Figure 3(c)). These data suggested that BRM270 inhibits efficiently the selfrenewal capacity of CD $44^{+}$PDAC cells via downregulation of stem cell factors.

3.4. BRM270 Restrains CD44 ${ }^{+}$PDAC Derived Tumor Growth via Sonic Hedgehog Signaling. Our previous studies showed antitumor effects of BRM270 in lung cancer and glioblastoma while there are no changes of body weight loss or side effects [13-15]. In this study, we examined whether BRM270 can suppress tumorigenesis derived $\mathrm{CD} 44^{+}$PDAC cells. After 5 weeks of inoculation, tumors treated with $5 \mathrm{mg} / \mathrm{kg}$ BRM 270 were significantly reduced as compared to PBS-treatment group (Figures 4(a) and 4(b)). Regarding signaling pathway, BRM270 downregulated Shh/Glil expressions, leading to the suppression of N-cad and MMP9 expression (Figure 4(c)). Moreover, there was dephosphorylation of STAT3, ERK1/2, and Akt downstream signaling pathways after exposure to $50 \mu \mathrm{g} / \mathrm{mL}$ BRM270 (Figure 4(d)). These data suggested that BRM270 suppresses the CD $44^{+}$PDAC cells derived tumor growth via Sonic hedgehog signaling pathway.

\section{Discussion}

Pancreatic cancer often has poor prognosis, and PDAC accounts for $85 \%$ in most pancreatic cancer diagnosed cases [19]. CD44 existence implies the EMT in pancreatic cancer, cancer stem cells, or drug resistance [6]. Therapies targeting CD44 show their efficiencies in prevention of cancer [6, $7,20]$. Our study indicated that BRM270 presents strong 

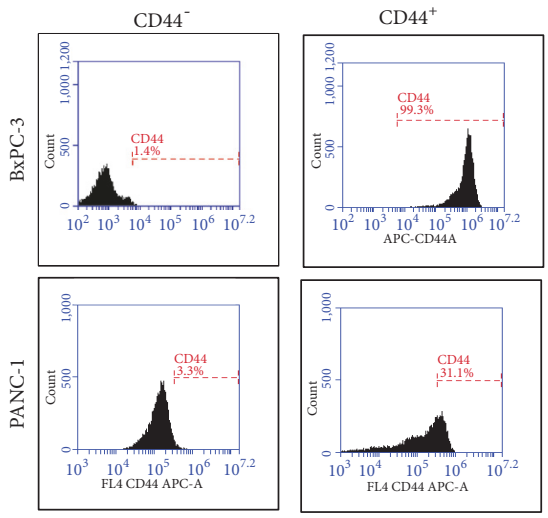

(a)
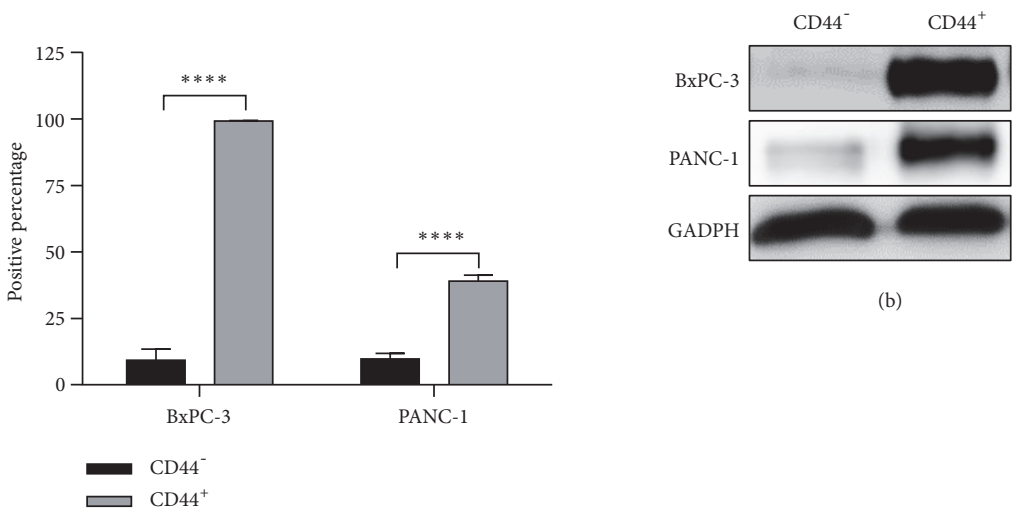

(b)
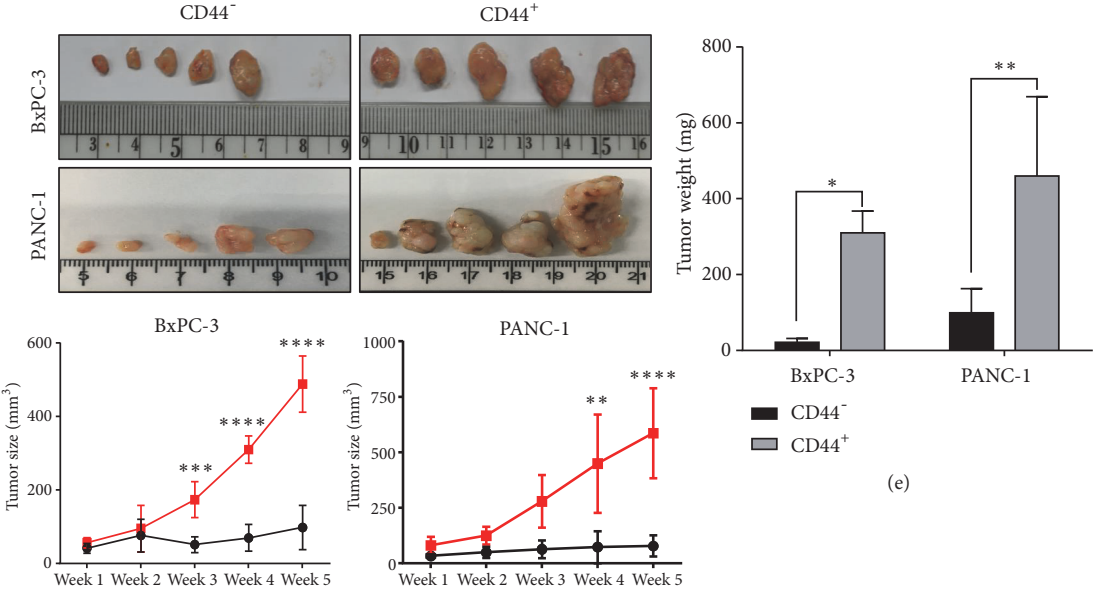

(e)
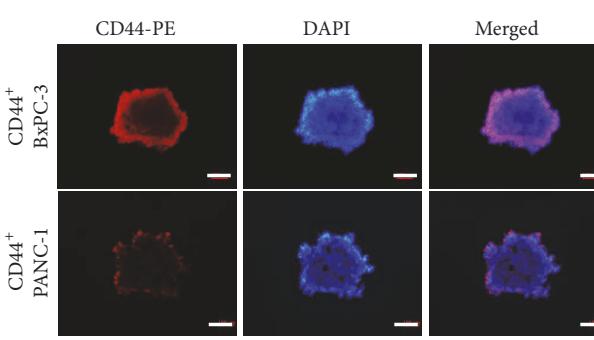

(c)

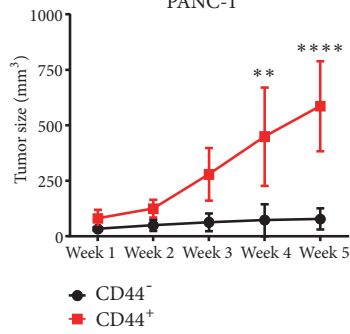

$\square \mathrm{CD} 44^{+}$

- $\mathrm{CD} 44^{-}$

$=\mathrm{CD} 44$

(d)

FIgURE 1: Isolation of CD44 $4^{+}$PDAC cells. (a) FACS analysis of CD44 surface marker in positive and negative CD44 BxPC-3 and PANC-1 after MACS separation. (b) Western blotting of lysates after MACS separation. (b) Immunocytochemistry staining of CD44, scale bar $20 \mu \mathrm{m}$. (d) Comparison of CD44 $4^{+}$and CD $44^{-}$induced tumor sizes. (e) Tumor weights of CD44 ${ }^{+}$and CD44 PDAC cells. Significant differences of values are compared to values of negative and positive CD44 cells and marked as follows: $* P<0.05 ; * * P<0.01 ; * * * P<0.001 ; * * * * P<0.0001$.

inhibitory effect on $\mathrm{CD} 44^{+}$PDAC cells via induction of cancer cell death, inhibitions of migration, invasion, and wound healing behaviors. Furthermore, BRM270 exhibits its potential in antistemness factor expressions such as CD133, Sox-2, Nanog, or Oct4. BRM270 also inhibits SALL4 , which acts as an oncofetal stemness gene and governs the expressions of stem cell factors and CD44 [21], resulting in CD44 diminution. SALL-4 existence in PDAC cells is believed to facilitate metastatic characteristics, via reactive oxygen species (ROS) regulation [16]. Therefore, suppression of SALL- 4 by BRM270 would possibly decrease metastasized death cases.

Chemoresistance and early metastatic spread induced by pancreatic CSCs are severe, resulting in the failure of current treatments [22]. Any therapies conquering chemoresistance and metastasis would efficiently advance antipancreatic cancer. Shh signaling is activated highly and involves notably such characteristics [10]. Inhibition of Shh signaling pathway restores chemosensitivity and frustrates self-renewal capacity in pancreatic CSCs $[11,12]$. In this study, BRM270 significantly suppresses Shh/Glil signaling pathway, leading to the downregulations of EMT genes, and consequently inhibits metastatic phenotype in CD $44^{+}$PDAC cells. Therefore, BRM270 could be the new adjuvant, partly sensitizing pancreatic CSCs to chemo-drugs while directly hitting selfrenewal capacity or metastatic spread.

At present, anti-PDAC by gemcitabine treatment recently shows resistance [8]. Furthermore, gemcitabine is believed to promote immunosuppressive tumor environment or invasiveness in pancreatic cancer [23, 24]. Therefore, minimizing gemcitabine dose by combination with other synergizing drugs or finding new replacement of chemotherapies is urgent. In this study, we selected two cell lines, the most sensitive and resistant to gemcitabine BxPC-3 and PANC-1, corresponding to wild type and mutant K-RAS, to investigate BRM270 effects. Results show that K-RAS with wild type 


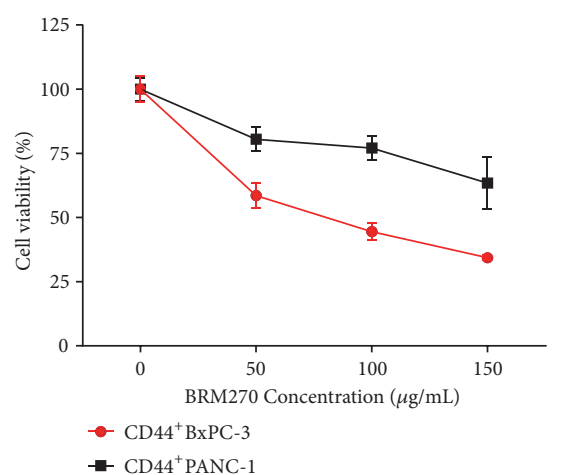

(a)

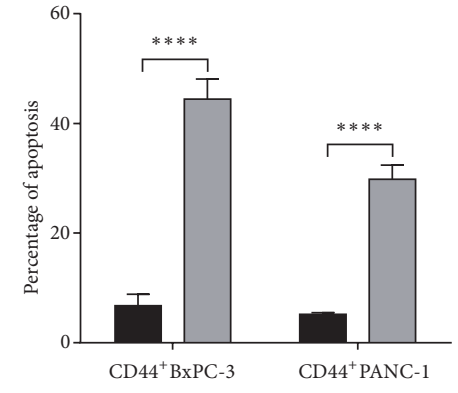

(-) BRM270 (+) BRM270

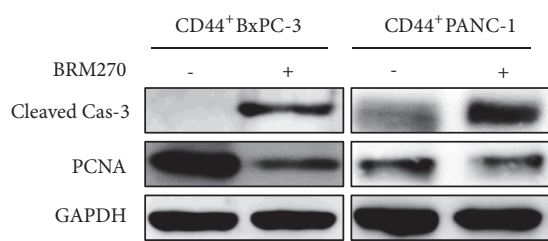

(c) (b)

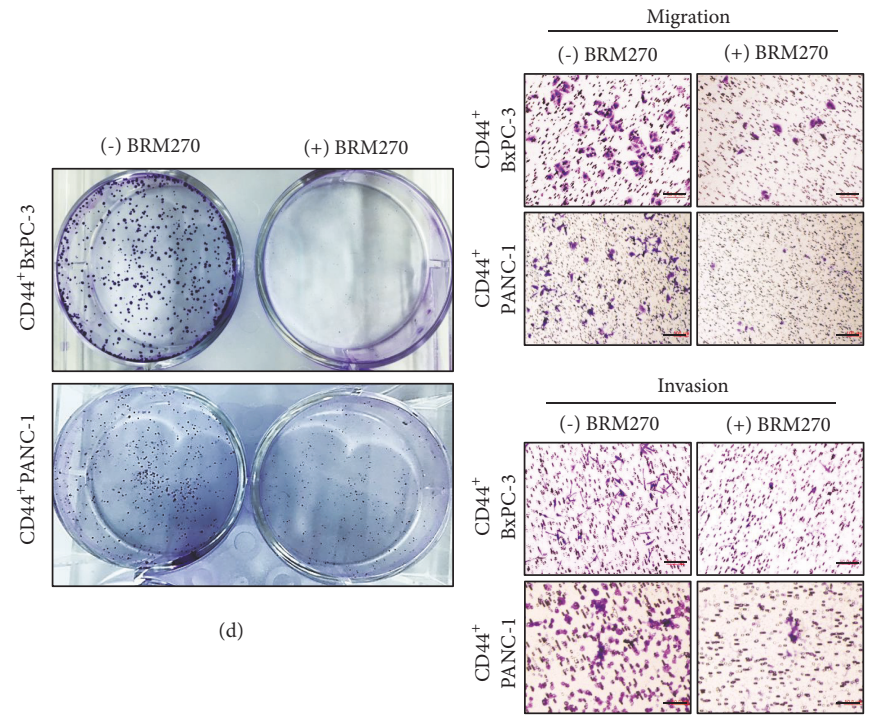

(e)
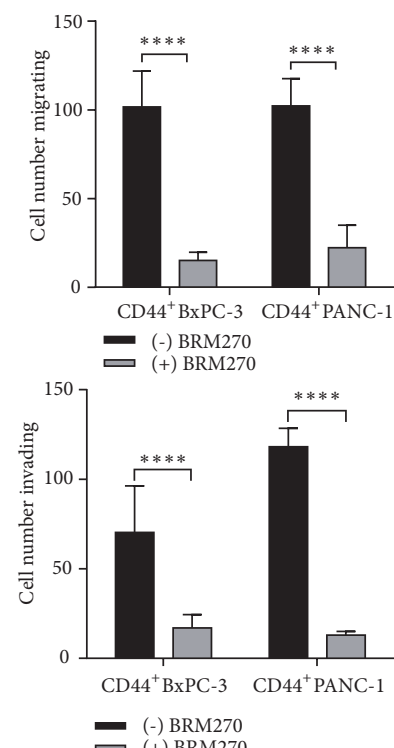

(+) BRM270

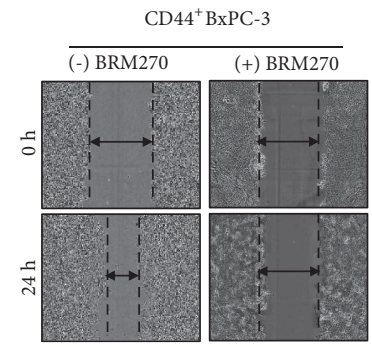

CD $44^{+}$PANC- 1

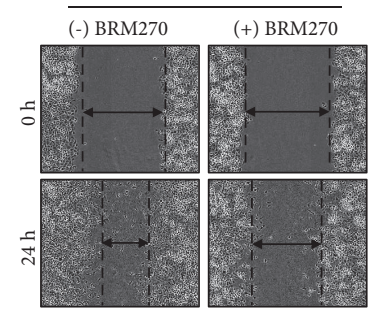

(f)

Figure 2: BRM270 suppresses the malignance of CD44 ${ }^{+}$PDAC cells. (a) Dose-dependent inhibitory effects of BRM270 to CD44 ${ }^{+}$PDAC cells. (b) The apoptosis of CD $44^{+}$PDAC cells exposed by BRM270, detected by FACS Annexin V. (c) Western blotting of apoptotic proteins in lysates with and without $50 \mu \mathrm{g} / \mathrm{mL}$ BRM270 treatment. (d) Clonogenic assay of CD44 ${ }^{+}$PDAC cells with and without $50 \mu \mathrm{g} / \mathrm{mL}$ BRM270 treatment. (e) Migration and invasion assay of CD $44^{+}$PDAC cells with and without $50 \mu \mathrm{g} / \mathrm{mL}$ BRM270 treatment, scale bar $100 \mu \mathrm{m}$. (f) Wound healing assay of CD $44^{+}$PDAC cells with and without $50 \mu \mathrm{g} / \mathrm{mL}$ BRM270 treatment. Significant differences of values are compared to values of positive CD44 cells with and without BRM270 treatment and marked as follows: $* * * * P<0.0001$.

(BxPC-3) responds sensitively to BRM270 as compared to the mutant (PANC-1). This event is similar to gemcitabine responses from these cell lines [25]. Possibility is that K-RAS mutant might subconsciously activate downstream signaling pathways including Akt or ERK, eventually resulting in cell survival [26].

Another possibility might be that the sensitivity to gemcitabine or BRM270 related to p53 status. BxPC-3 and PANC-1 have mutant TP53 at Y220C and R273H, respectively. Y220C is believed to destabilize p53 while $\mathrm{R} 273 \mathrm{H}$ affects p53 binding function [27]. BRM270 might help stabilize p53 conformation, resulting in more wild type p53, and consequently drives apoptosis once treatment occurs. Therefore, BxPC-3 with Y220C TP53 is sensitive to BRM270. Generally, BRM270 in our study inhibits both K-RAS mutant and wild type or TP53 mutant activities by suppressing Akt and ERK1/2 or
STAT3 phosphorylation signaling, presenting its extensive inhibitory effects on the main mutations causing PDAC. Definitely, there are many dark sides needed to be uncovered, such as the effects of BRM270 on tumor environment at early metastasis stage or its behaviors in anti-metastasizing or anti-circulating pancreatic tumor cells or the combination between BRM270 and gemcitabine in pancreatic cancer intervention. Nevertheless, these findings initiatively assert BRM270 uses in attempts of antipancreatic cancer, the first step for safe therapies.

\section{Data Availability}

The data used to support the findings of this study are available from the corresponding author upon request. 


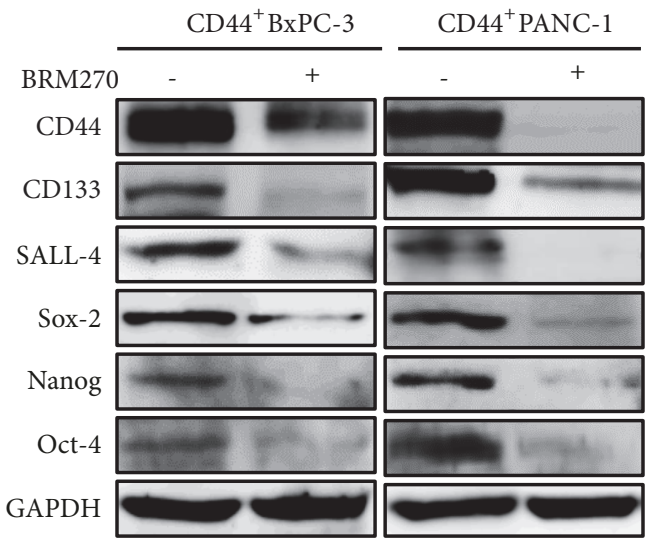

(a)

)

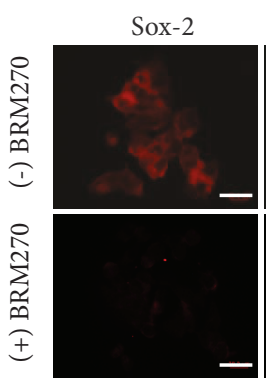

Oct4

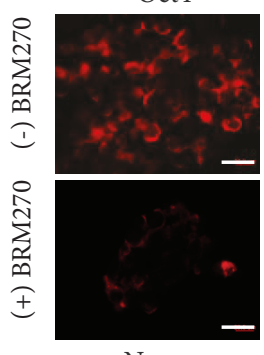

Nanog
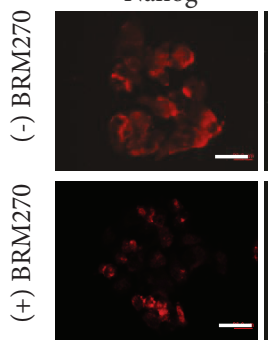

I

(-) BRM270

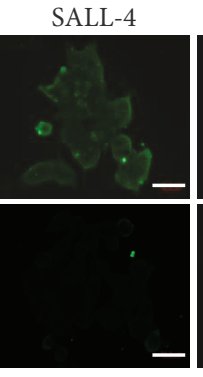

DAPI
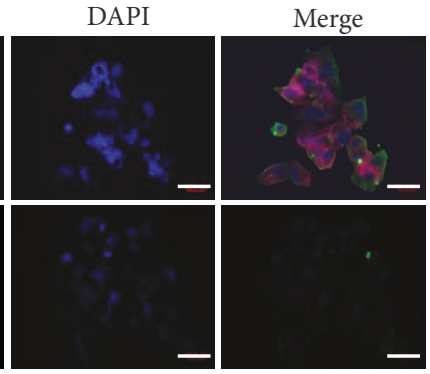

$\mathrm{CD} 44$

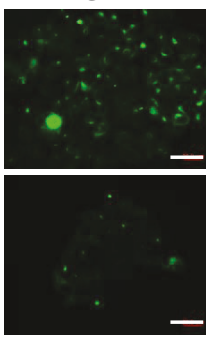

DAPI

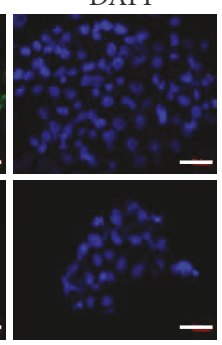

Merge

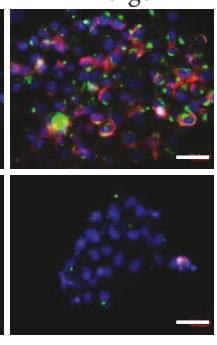

CD133

DAPI
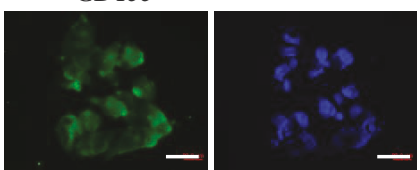

Merge

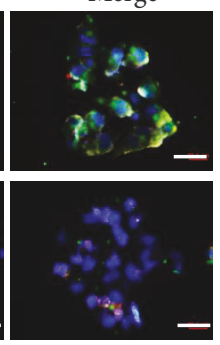

(b)

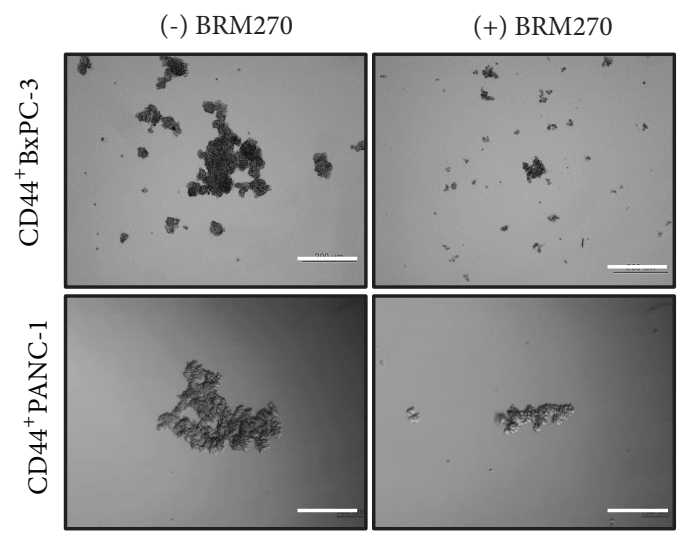

(c)

FIGURE 3: BRM270 inhibits self-renewal capacity of CD44+ PDAC cells. (a) Western blotting of stemness genes in lysates treated with and without $50 \mu \mathrm{g} / \mathrm{mL}$ BRM270. (b) Immunocytochemistry staining of stemness genes in CD $44^{+}$PDAC cells with and without $50 \mu \mathrm{g} / \mathrm{mL}$ BRM270 treatment, scale bar $20 \mu \mathrm{m}$. (c) Sphere formation assay of CD $44^{+}$PDAC cells with and without $50 \mu \mathrm{g} / \mathrm{mL}$ BRM270 treatment, scale bar 100 $\mu \mathrm{m}$.

\section{Conflicts of Interest}

The authors declare no conflicts of interest.

\section{Authors' Contributions}

Do Luong Huynh and Hyebin Koh contributed equally to the manuscript.

\section{Acknowledgments}

This research was financially supported by the Ministry of Trade, Industry, and Energy (MOTIE), Korea, under the "Regional Specialized Industry Development Program (R\&D, P0002062)" supervised by Korea Institute for Advancement of Technology (KIAT). 

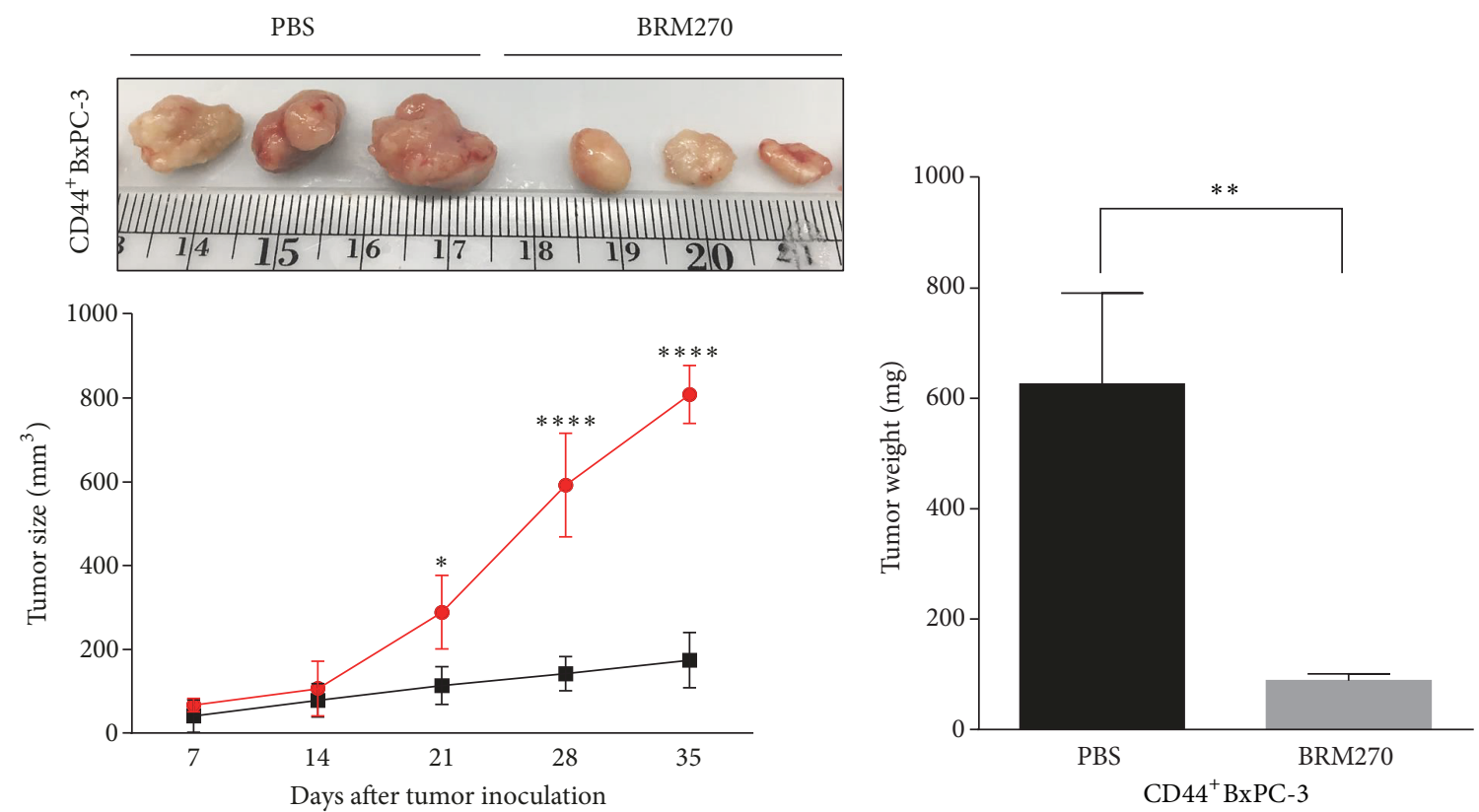

(a)

(b)

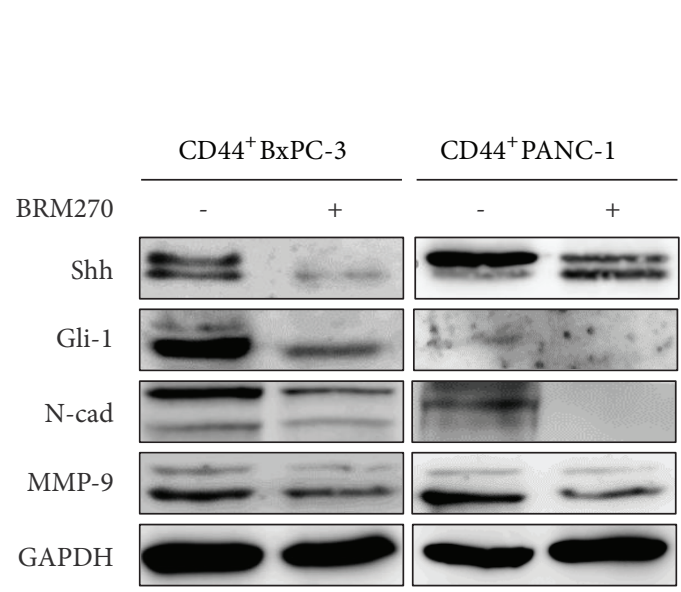

(c)

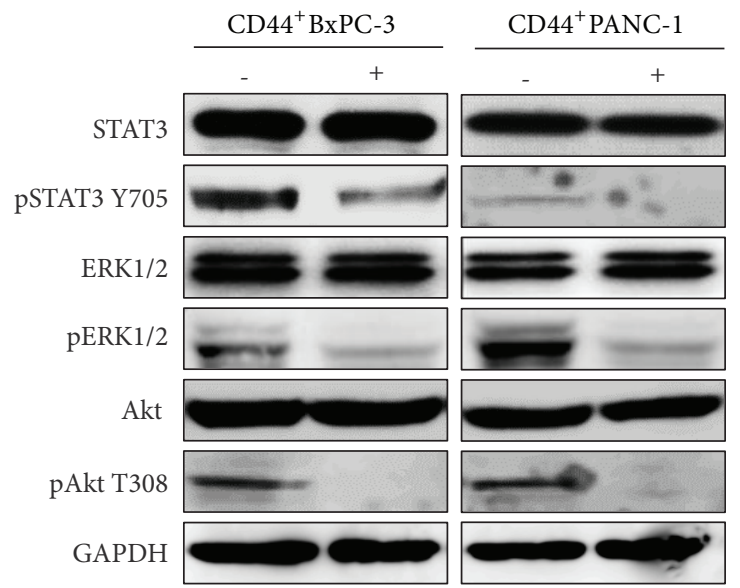

(d)

Figure 4: BRM270 restrains CD44 PDAC cells derived tumor growth via downregulating Sonic hedgehog signaling pathway. (a) Sizes of tumors with and without $5 \mathrm{mg} / \mathrm{kg}$ BRM270 treatment after 35-day inoculation. (b) Weights of tumors with and without $5 \mathrm{mg} / \mathrm{kg}$ BRM270 treatment. (c) Western blotting of epithelial-mesenchymal transition (EMT) genes in lysates treated with and without BRM270 $50 \mu \mathrm{g} / \mathrm{mL}$. (d) Western blotting of signaling pathways in lysates treated with and without BRM270 $50 \mu \mathrm{g} / \mathrm{mL}$. Significant differences of values are compared to values of groups treated and nontreated with BRM270 and marked as follows: $* P<0.05 ; * * P<0.01 ; * * * * P<0.0001$.

\section{References}

[1] O. P. Zakharova, G. G. Karmazanovsky, and V. I. Egorov, "Pancreatic adenocarcinoma: outstanding problems," World Journal of Gastrointestinal Surgery, vol. 4, no. 5, pp. 104-113, 2012.

[2] T. Muniraj, P. A. Jamidar, and H. R. Aslanian, "Pancreatic cancer: a comprehensive review and update," Disease- $a-$ Month, vol. 59, no. 11, pp. 368-402, 2013.

[3] S. Yachida, S. Jones, I. Bozic et al., "Distant metastasis occurs late during the genetic evolution of pancreatic cancer," Nature, vol. 467, no. 7319, pp. 1114-1117, 2010.
[4] A. Mohammed, N. B. Janakiram, S. Lightfoot, H. Gali, A. Vibhudutta, and C. V. Rao, "Early detection and prevention of pancreatic cancer: Use of genetically engineered mouse models and advanced imaging technologies," Current Medicinal Chemistry, vol. 19, no. 22, pp. 3701-3713, 2012.

[5] H. Xu, Y. Tian, X. Yuan et al., "The role of CD44 in epithelial-mesenchymal transition and cancer development," OncoTargets and Therapy, vol. 8, pp. 3783-3792, 2015.

[6] C. Chen, S. Zhao, A. Karnad, and J. W. Freeman, "The biology and role of CD44 in cancer progression: Therapeutic implications," Journal of Hematology \& Oncology, vol. 11, no. 1, 2018. 
[7] E. Dalla Pozza, C. Lerda, C. Costanzo et al., "Targeting gemcitabine containing liposomes to CD44 expressing pancreatic adenocarcinoma cells causes an increase in the antitumoral activity," Biochimica et Biophysica Acta (BBA) - Biomembranes, vol. 1828, no. 5, pp. 1396-1404, 2013.

[8] M. Amrutkar and I. P. Gladhaug, "Pancreatic cancer chemoresistance to gemcitabine," Cancers, vol. 9, no. 11, article no. 157, 2017.

[9] S.-N. Tang, J. Fu, D. Nall, M. Rodova, S. Shankar, and R. K. Srivastava, "Inhibition of sonic hedgehog pathway and pluripotency maintaining factors regulate human pancreatic cancer stem cell characteristics," International Journal of Cancer, vol. 131, no. 1, pp. 30-40, 2012.

[10] H. Onishi and M. Katano, "Hedgehog signaling pathway as a new therapeutic target in pancreatic cancer," World Journal of Gastroenterology, vol. 20, no. 9, pp. 2335-2342, 2014.

[11] S. Nagai, M. Nakamura, K. Yanai et al., "Glil contributes to the invasiveness of pancreatic cancer through matrix metalloproteinase-9 activation," Cancer Science, vol. 99, no. 7, pp. 1377-1384, 2008.

[12] M. Mimeault and S. K. Batra, "Frequent deregulations in the hedgehog signaling network and cross-talks with the epidermal growth factor receptor pathway involved in cancer progression and targeted therapies," Pharmacological Reviews, vol. 62, no. 3 , pp. 497-524, 2010.

[13] R. K. Mongre, S. S. Sodhi, N. Sharma et al., "Epigenetic induction of epithelial to mesenchymal transition by LCN2 mediates metastasis and tumorigenesis, which is abrogated by NF-B inhibitor BRM270 in a xenograft model of lung adenocarcinoma," International Journal of Oncology, vol. 48, no. 1, pp. 84-98, 2016.

[14] H.-Y. Jeon, C. G. Park, S. W. Ham et al., "BRM270, a compound from natural plant extracts, inhibits glioblastoma stem cell properties and glioblastoma recurrence," Journal of Medicinal Food, vol. 20, no. 9, pp. 838-845, 2017.

[15] T. Kwon, N. Chandimali, D. L. Huynh et al., "BRM270 inhibits cancer stem cell maintenance via microRNA regulation in chemoresistant A549 lung adenocarcinoma cells article," Cell Death \& Disease, vol. 9, no. 2, p. 244, 2018.

[16] D. L. Huynh, J. J. Zhang, N. Chandimali et al., "SALL4 suppresses reactive oxygen species in pancreatic ductal adenocarcinoma phenotype via FoxM1/Prx III axis," Biochemical and Biophysical Research Communications, vol. 503, no. 4, pp. 22482254, 2018.

[17] C. Hadjimichael, K. Chanoumidou, N. Papadopoulou, P. Arampatzi, and J. Papamatheakis, "Kretsovali A: common stemness regulators of embryonic and cancer stem cells," World Journal of Stem Cells, vol. 7, no. 9, pp. 1150-1184, 2015.

[18] H. Tatetsu, N. R. Kong, G. Chong, G. Amabile, D. G. Tenen, and L. Chai, "SALL4, the missing link between stem cells, development and cancer," Gene, vol. 584, no. 2, pp. 111-119, 2016.

[19] A. F. Hezel, A. C. Kimmelman, B. Z. Stanger, N. Bardeesy, and R. A. Depinho, "Genetics and biology of pancreatic ductal adenocarcinoma," Genes \& Development, vol. 20, no. 10, pp. 1218-1249, 2006.

[20] S. Zhao, C. Chen, K. Chang et al., "CD44 expression level and isoform contributes to pancreatic cancer cell plasticity, invasiveness, and response to therapy," Clinical Cancer Research, vol. 22, no. 22, pp. 5592-5604, 2016.

[21] X. Yuan, X. Zhang, W. Zhang et al., "SALL4 promotes gastric cancer progression through activating CD44 expression," Oncogenesis, vol. 5, no. 11, p. e268, 2016.
[22] P. C. Hermann and B. Sainz, "Pancreatic cancer stem cells: A state or an entity?" Seminars in Cancer Biology, 2018.

[23] S. K. Deshmukh, N. Tyagi, M. A. Khan et al., "Gemcitabine treatment promotes immunosuppressive microenvironment in pancreatic tumors by supporting the infiltration, growth, and polarization of macrophages," Scientific Reports, vol. 8, no. 1, p. 12000, 2018.

[24] S. Arora, A. Bhardwaj, S. Singh et al., "An undesired effect of chemotherapy: Gemcitabine promotes pancreatic cancer cell invasiveness through reactive oxygen species-dependent, nuclear factor $\kappa b$ - and hypoxia-inducible factor $1 \alpha$-mediated up-regulation of CXCR4," The Journal of Biological Chemistry, vol. 288, no. 29, pp. 21197-21207, 2013.

[25] Y. Kim, D. Han, H. Min, J. Jin, E. C. Yi, and Y. Kim, "Comparative proteomic profiling of pancreatic ductal adenocarcinoma cell lines," Molecules and Cells, vol. 37, no. 12, pp. 888-898, 2014.

[26] B. Zhao, L. Wang, H. Qiu et al., "Mechanisms of resistance to anti-EGFR therapy in colorectal cancer," Oncotarget, vol. 8, no. 3, pp. 3980-4000, 2017.

[27] W. A. Freed-Pastor and C. Prives, "Mutant p53: one name, many proteins," Genes \& Development, vol. 26, no. 12, pp. 1268-1286, 2012. 


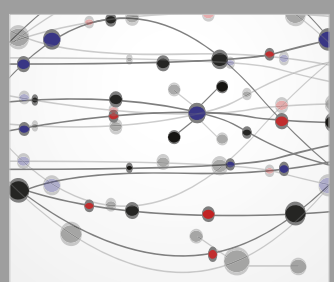

The Scientific World Journal
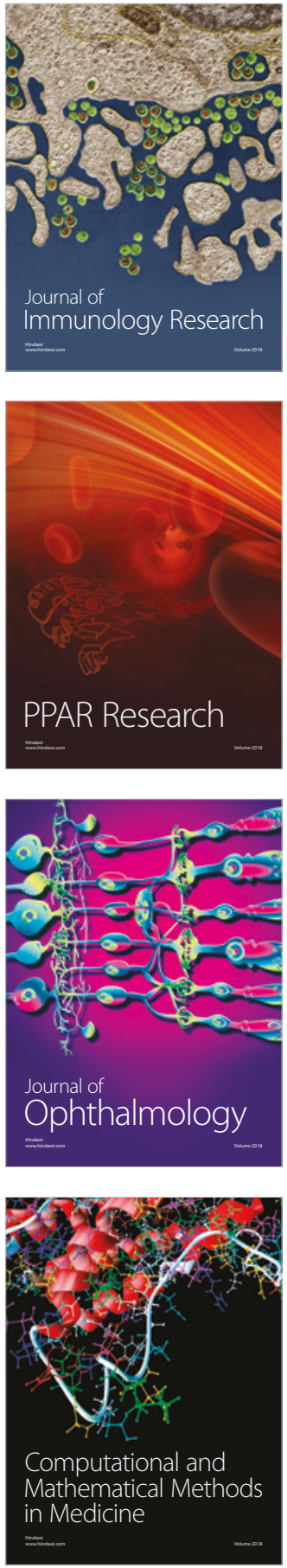

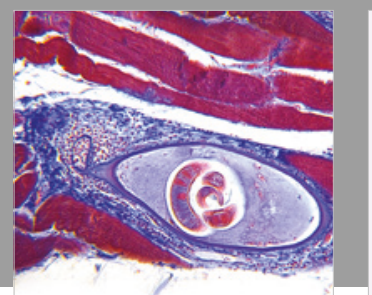

Gastroenterology Research and Practice

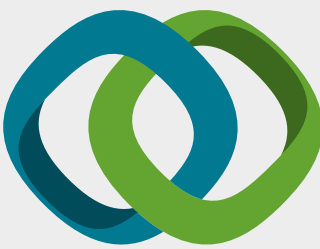

\section{Hindawi}

Submit your manuscripts at

www.hindawi.com
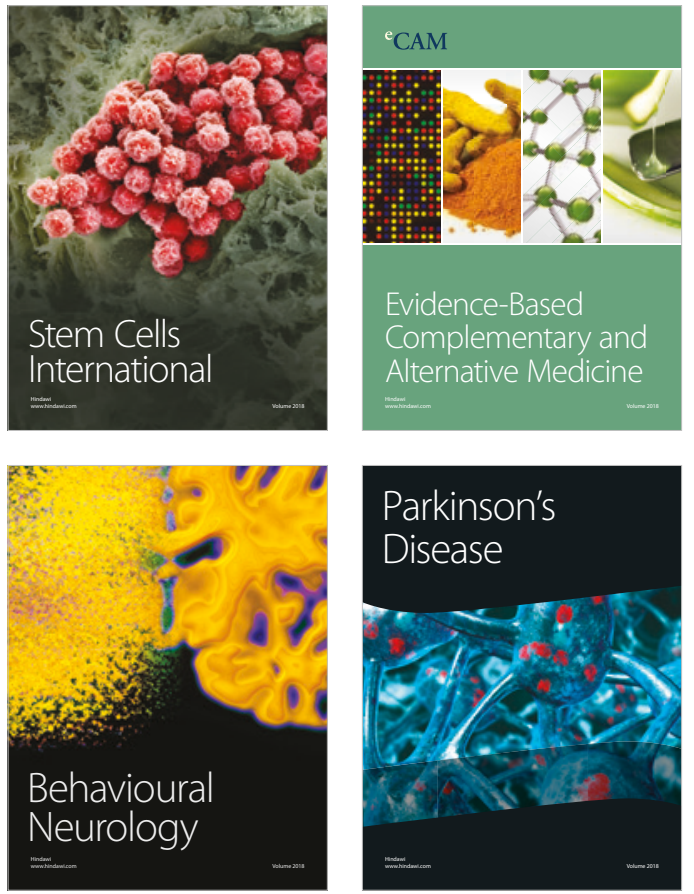

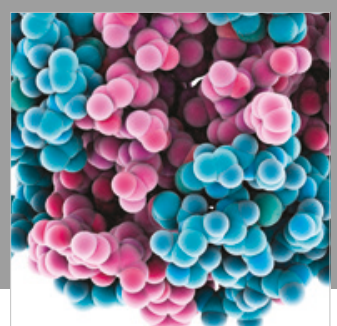

ournal of

Diabetes Research

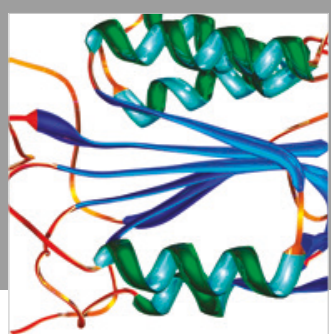

Disease Markers
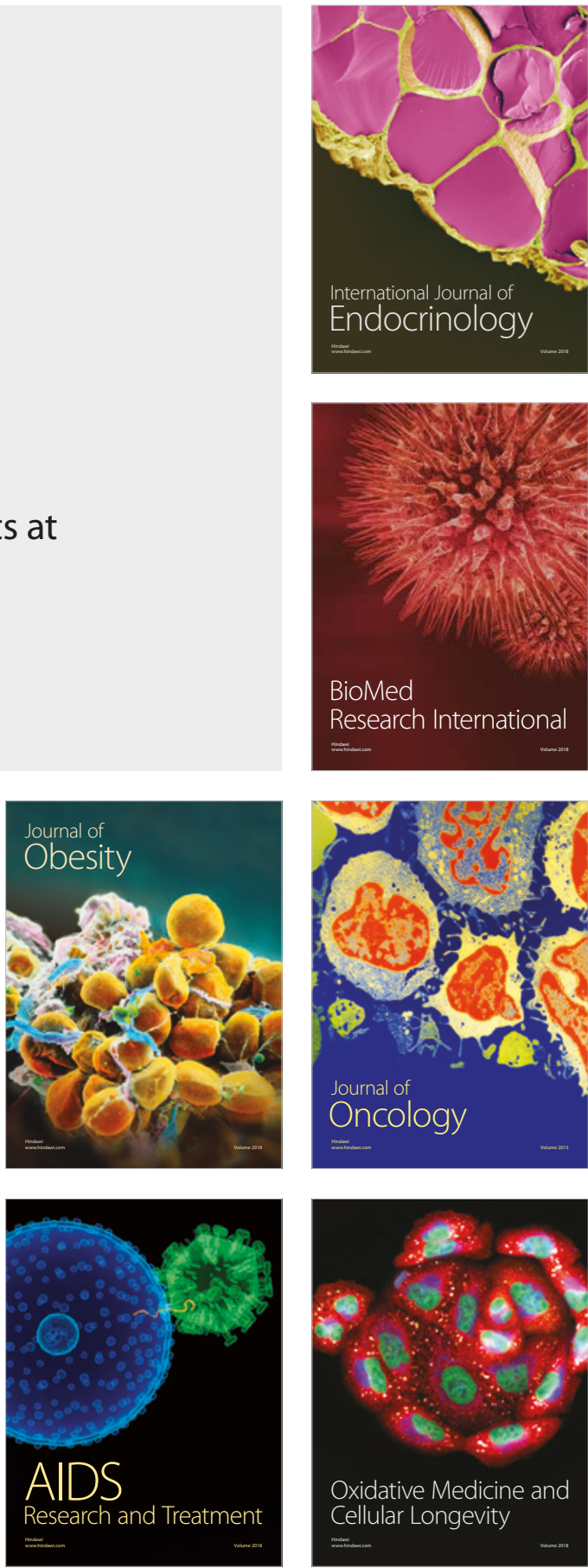